\title{
Hard X-ray Phase-Contrast Tomographic Nanoimaging
}

\author{
M. Stampanoni ${ }^{\mathrm{a}, \mathrm{b}}$, F. Marone ${ }^{\mathrm{a}}$, J. Vila-Comamala ${ }^{\mathrm{a}}$, S. Gorelick ${ }^{\mathrm{a}}$, \\ C. David ${ }^{\mathrm{a}}$, P. Trtik ${ }^{\mathrm{c}}$, K. Jefimovs ${ }^{\mathrm{c}}$, and R. Mokso ${ }^{\mathrm{a}}$ \\ ${ }^{a}$ Paul Scherrer Institut, 5232 Villigen, Switzerland \\ ${ }^{b}$ Institute for Biomedical Engineering, University and ETH Zürich, 8092 Zürich, Switzerland \\ ${ }^{c}$ EMPA, Swiss Federal Laboratories for Materials Science and Technology, 8600 Dübendorf, Switzerland
}

\begin{abstract}
Synchrotron-based full-field tomographic microscopy established itself as a tool for noninvasive investigations. Many beamlines worldwide routinely achieve micrometer spatial resolution while the isotropic 100-nm barrier is reached and trespassed only by few instruments, mainly in the soft x-ray regime. We present an x-ray, full-field microscope with tomographic capabilities operating at $10 \mathrm{keV}$ and with a 3D isotropic resolution of $144 \mathrm{~nm}$ recently installed at the TOMCAT beamline of the Swiss Light Source. Custom optical components, including a beam-shaping condenser and phase-shifting dot arrays, were used to obtain an ideal, aperture-matched sample illumination and very sensitive phase-contrast imaging. The instrument has been successfully used for the nondestructive, volumetric investigation of single, unstained cells.
\end{abstract}

Keywords: Full field microscopy, nanotomography, x-ray imaging, synchrotron CT, phase-contrast imaging PACS: 87.59.-e, 68.37.Yz, 07.85.Tt, 87.64.mh

\section{INTRODUCTION}

Most of synchrotron-based tomographic microscopy end stations installed worldwide are based on parallel-beam geometry, the micrometer resolution limit being then essentially given by the spatial resolution of the detector. To reach and trespass the isotropic barrier of $100 \mathrm{~nm}$, however, the use of magnifying x-ray optics is mandatory. This challenge has been tackled efficiently by only a few instruments worldwide, mostly in the soft x-ray range [1,2]. When imaging biological samples, $x$-rays within the water window (between $283 \mathrm{eV}$ and $530 \mathrm{eV}$ ) generate highcontrast images because of the large photoelectrical cross-section difference between the carbon-based structures and the surrounding aqueous medium. However, the short focal length of Fresnel zone plates (used as microscope optics) within this energy range combined with the inherent strong absorption of air along the beam path, results in complex instrumental equipment and unpractical experimental conditions, especially when the sample needs to be cryo-cooled in order to reduce radiation damage. Moreover, the usage of soft x-ray microscopes is problematic for thick specimens, as the penetration capability is low and the small depth of focus limits the resolution. Operating a microscope at hard $\mathrm{x}$-rays could solve part of these problems since the higher penetration depth of the radiation, together with the larger depth-of-focus of the optical components, would allow for the investigation of thicker samples relaxing the experimental conditions. At hard x-ray energies, however, biological samples-consisting mainly of low-Z elements - exhibit a small photoelectric cross section, resulting in a poor absorption signal and making the use of phase contrast unavoidable. This paper presents some results from a recently developed instrument [3] that efficiently records tomographic absorption and phase-contrast images at measured 3D spatial resolution of $144 \mathrm{~nm}$.

\section{INSTRUMENTATION}

One of the most common approaches to efficiently achieve sub-100-nm resolution is full-field microscopy. The key optical elements are a condenser, to provide illumination of the sample, and an objective lens, to produce a magnified image of the sample on the detector. The ideal illumination should be as homogeneous and as intense as

The 10th International Conference on X-ray Microscopy

AIP Conf. Proc. 1365, 239-242 (2011); doi: 10.1063/1.3625348

(C) 2011 American Institute of Physics 978-0-7354-0925-5/\$30.00 
possible and its numerical aperture should be matched to that of the objective lens in order to obtain optimum resolution. Condensing x-rays with Fresnel zone plates (FZPs) [4], tapered capillaries [5], mirrors [6] or combinations of these devices [7] is a common solution. These optics focus the beam into a Gaussian-shaped spot, normally smaller than the field of view of the microscope, which therefore requires the condenser to be scanned along the direction transverse to the beam to partially overcome this inhomogeneous illumination. Beamshapers with complex functionality - essentially a conventional FZP divided into sectors while keeping local spatial frequency within each sector constant — have been suggested as a possible alternative to provide ideal illumination conditions. At the beamline for Tomographic Microscopy and Coherent Radiology Experiments (TOMCAT) of the Swiss Light Source [8], we installed a beamshaper that provides a square top-flat illumination $(50 \times 50$ microns, see Fig. 1(c) $)$ at the sample position [9]. Each subfield consists of a linear grating with constant line orientation and period both corresponding to the local orientation and period of the structures of the FZP.

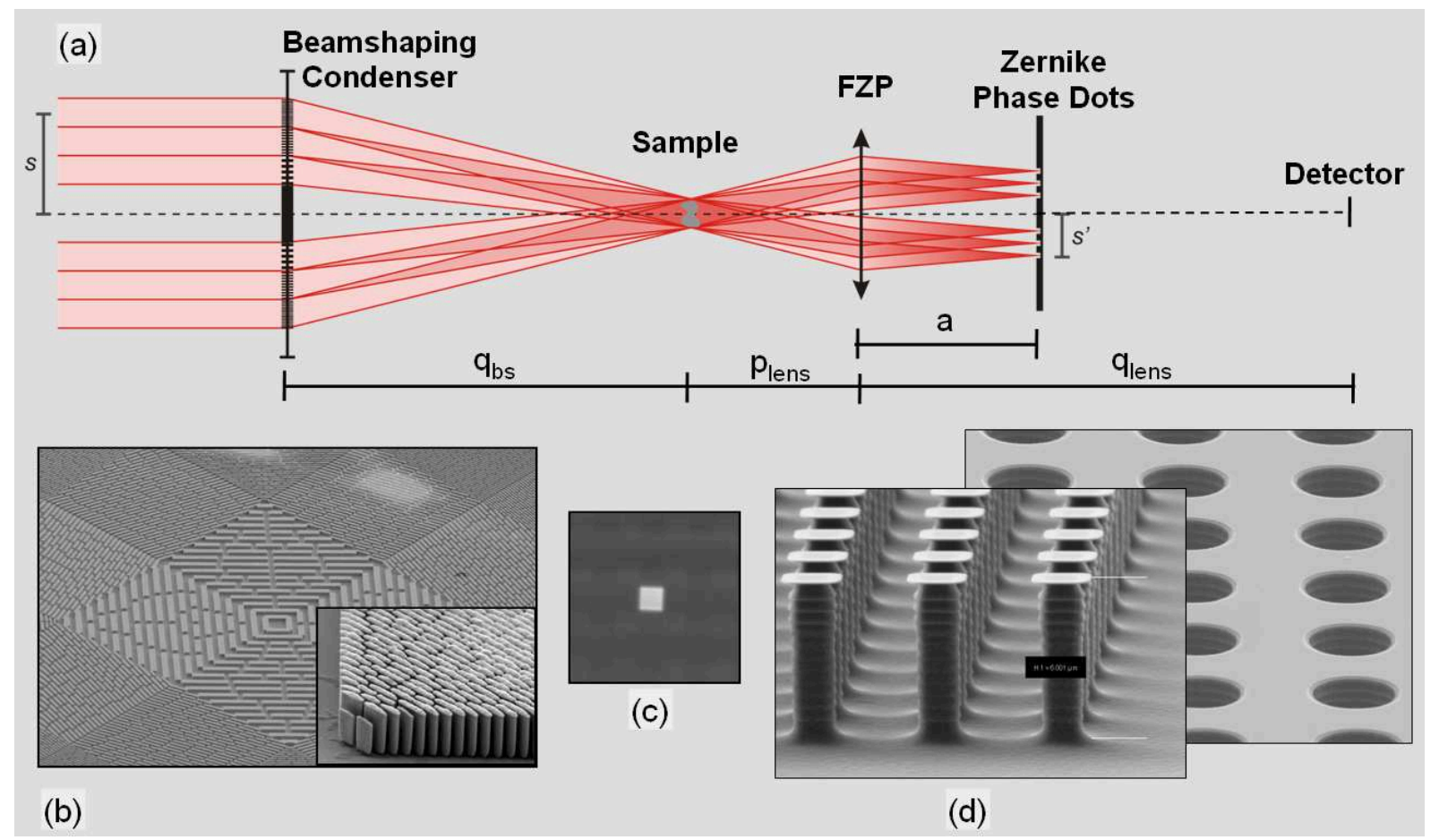

FIGURE 1. (a) Schematic representation of the experimental setup. The beamshaper (b) diffracts the incoming radiation down to a $50 \times 50 \mu^{2}$ top-flat square spot (c). The wave front is manipulated in the back-focal plane of the magnifying zone plate. Either pillars ((d), foreground) or dots ((d), background) can be used to shift the phase (by $\pi / 2$ for instance) of the diffracted beam with respect to the undiffracted one, generating Zernike phase contrast.

The layout of the experiment is described in Fig. 1. The beamshaper is $1 \times 1 \mathrm{~mm}^{2}$ in size, with subfields of $50 \times$ $50 \mu \mathrm{m}^{2}$ and 1- $\mu \mathrm{m}$-thick gold structures, see Fig. 1(b) and Gorelick et al. [10]. The outermost zone width is $\Delta \mathrm{r}_{\mathrm{bs}}=100$ $\mathrm{nm}$, see inset of Fig. 1(b). At $10 \mathrm{keV}$ this yields a focal length of $\mathrm{f}_{\mathrm{bs}}=1083 \mathrm{~mm}$. A square central stop $(600 \times 600$ $\mu \mathrm{m}^{2}$ ) has been used to block the zeroth order, and the size of its shadow is matched to the size of the beamshaper illumination magnified by the objective lens on the detector. As objective lens, we used a gold FZP, obtained by electroplating into a polyimide mould [11], with an outermost zone width of $\Delta \mathrm{r}=100 \mathrm{~nm}$, a zone height of $1 \mu \mathrm{m}$, a diameter of $100 \mu \mathrm{m}$, and a focal length of $\mathrm{f}_{\text {lens }}=80.65 \mathrm{~mm}$. For a distance between lens and detector of approximately $9000 \mathrm{~mm}$, this results in a magnification factor of $\mathrm{M}=110 \times$. Each sector of the beamshaper produces a spot in the back-focal plane of the objective lens. The resolution of the system has been measured according to Modregger et al. [12]: we obtained a value of $133 \mathrm{~nm}$ for 2D images and $144 \mathrm{~nm}$ for 3D images, i.e. very close to the Rayleigh limit of $R_{\text {Rayleigh }}=1.22 \cdot \Delta r=122 \mathrm{~nm}$. The images have been oversamples twice, i.e., the voxel size was $\sim 60 \mathrm{~nm}$.

Phase contrast is obtained by manipulating the wavefront in the back-focal plane of the magnifying zone plate, according to Zernike's principle. Traditionally, such systems are implemented using hollow cone illumination and 
an imaging objective in combination with a separate $\pm \pi / 2$ phase ring [13]. In our case, due to the particular illumination geometry (an array of spots) at the back focal length, a phase ring will no longer work, and it must be replaced by a phase dots array or a phase pillars array, see Fig. 1(d). The depth of the dots - or the height of the pillars - can be adjusted to generate the desired phase shift. The use of phase dots — or pillars - instead of more conventional rings is imposed by the illumination geometry, but it brings an important advantage in terms of image quality. Using such discrete structures as phase shifting elements and being able to precisely control their size allow reduction of the "halo" effects, as suggested by Maurer et al. [14]. Further, compared to a conventional Zernicke setup, which is limited to the use of a narrow condenser ring collecting only a small fraction of the incoming radiation, our combination of beamshaper with phase-shifting dot arrays is much more photon-efficient, as it accepts a large fraction of the incoming flux. Additional details about the instrumentation can be found in Stampanoni et al. [3].

\section{RESULTS}

Our instrument can be operated either in absorption or in phase-contrast mode. Switching between both modalities is done by simply positioning the phase dots (or pillar) either away from the optical axis (absorption) or on the optical axis (phase). Figures 2(a)-(c) show the radiographic projections of tiny polystyrene spheres (diameter varying from 2 to 5 microns) acquired either with the phase dots out of the beam path (Fig. 2(a)) or in the beam path (Figs. 2(b), 2(c)). All three images have been recorded at $10 \mathrm{keV}$. At such energy, the spheres are almost ideal phase objects, and therefore they are not visible when the microscope is operated in absorption mode. Depending on the position of the phase dots with respect to the diffraction maxima, it is possible to generate either positive phase contrast (phase shift of $+\pi / 2$, Fig. 2(b)) or negative contrast (phase shift of $-\pi / 2$, Fig. 2(c)).

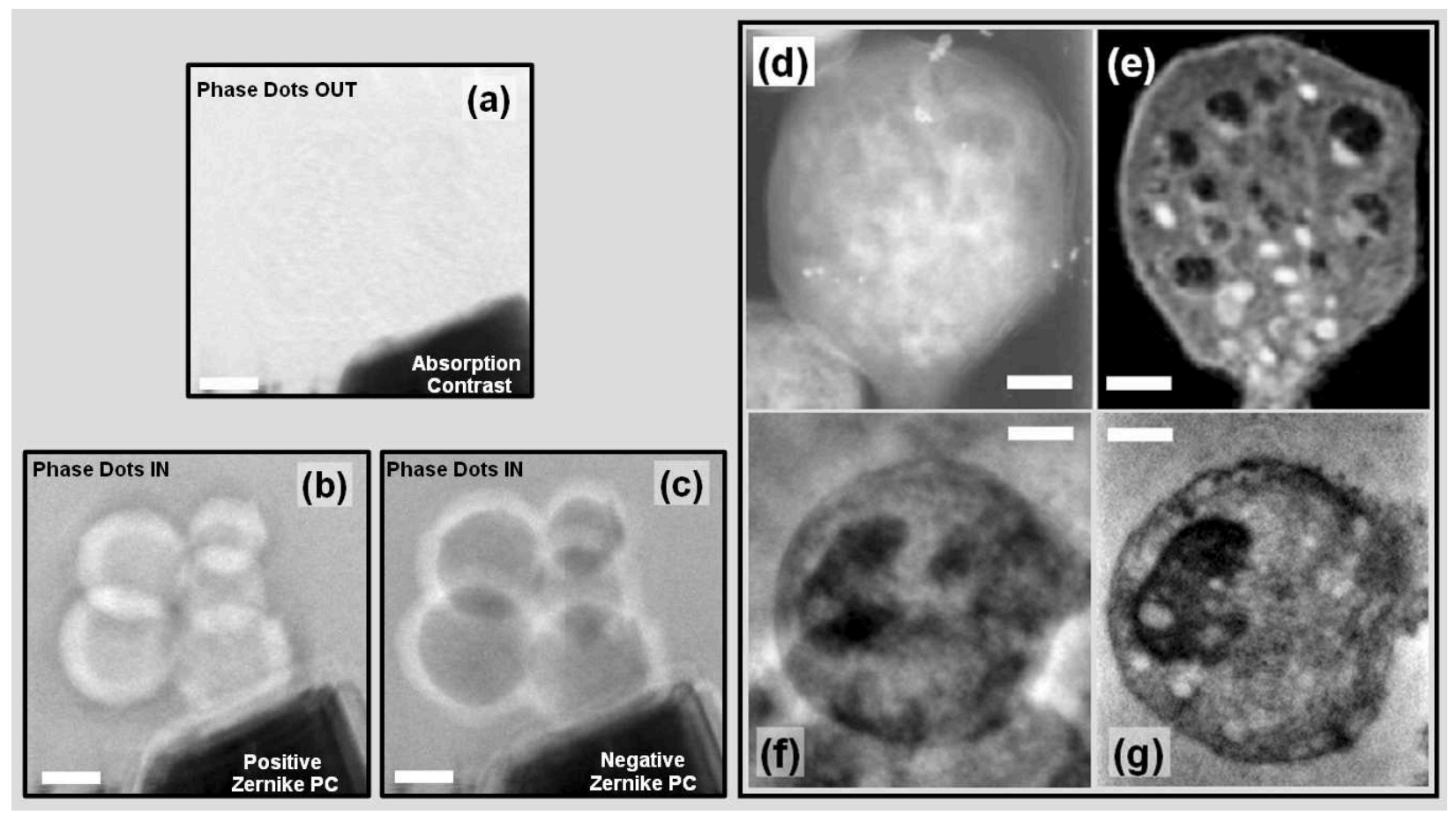

FIGURE 2. (a) Radiographic projection of a few polystyrene spheres in absorption mode (10 keV). (b, c) Positive resp. negative phase-contrast image of the same spheres. $(\mathrm{d}$, e) Absorption-based radiographic projection and tomographic slice through a yeast cell (adapted from Larabell et al. [15]) obtained with the XM-1 microscope operated in the water window. (f, g) Phase-contrast radiographic projection and tomographic slice through a MC3 preosteoblast cell obtained with the TOMCAT microscope (adapted from Stampanoni et al. [3]). Scale bars: (a-c) $2 \mu \mathrm{m}$, (d,e) $0.5 \mu \mathrm{m}$, (f,g) $5 \mu \mathrm{m}$.

In a more sophisticated experiment, we imaged MC3 preosteoblast cells—-fixed but not stained — contained in a glass capillary (100- $\mu \mathrm{m}$ diameter, $10-\mu \mathrm{m}$ wall thickness) and suspended in a $1.3 \%$ wt hydrogel. The system was very stable, and we could easily acquire a full tomographic data set (251 projections) in approximately 10 minutes. We would like to compare our radiographic projection (Fig. 2(f)) and tomographic reconstruction (Fig. 2(g)) with the 
well-known yeast cell radiography and tomography (Fig. 2(c), 2(d)) obtained by Larabell et al. [15] in absorption mode. Even though our microscope does not yet achieve the spatial resolution of the Berkeley XM-1 and XM-2 microscopes - we designed our system in order to reach isotropic resolution around $120 \mathrm{~nm}$ - it appears obvious that the sensitivity provided by phase contrast allows to visualize (both in radiography and tomography) very similar structures. It has to be noted that our samples have not been cryo-cooled since we did not observe particular radiation damage within the time (typically a few minutes) of the tomographic scan.

\section{CONCLUSIONS AND OUTLOOK}

We presented a full-field microscope that works both in absorption and phase-contrast regimes. In spite of the fact that the microscope is operated with broadband hard $\mathrm{x}$-rays $\left([\mathrm{Ru} / \mathrm{C}]_{100}\right.$ double-bounce multilayer monochromator), an isotropic spatial resolution of $144 \mathrm{~nm}$ has been reached, very close to the Rayleigh limit of 122 $\mathrm{nm}^{3}$. With the use of an alternative illumination design as well as an innovative, Zernike-based, phase-contrast approach, we demonstrated that it is possible to obtain high-quality radiographic and tomographic information on biological relevant samples, without the need of cryo-cooling and with a significant reduction of dose deposition compared to conventional, absorption-based microscopes.

\section{ACKNOWLEDGMENTS}

We greatly acknowledge G. Mikuljan of the SLS TOMCAT beamline staff for his fundamental support in setting up the experiment. We are also indebted to Dr. M. Feser of Xradia Inc. for valuable discussions on Zernike phase contrast.

\section{REFERENCES}

1. D. Weiss et al., Ultramicroscopy 84, 185 (2000).

2. W. Meyer-Ilse et al., J. Microsc. 201, 395 (2001).

3. M. Stampanoni et al., Phys. Rev. B 81, 140105 (2010).

4. E. H. Anderson et al., J. Vac. Sci. Technol. B 18, 2970 (2000).

5. G. C. Yin et al., Appl. Phys. Lett. 89, 221122 (2006).

6. C. Rau et al., IPAP Conf. Series 7, 7 (2005).

7. E. Di Fabrizio et al., Microelectron. Eng. 67-8, 87 (2003).

8. M. Stampanoni et al., AIP Conf. Proc 879, 848 (2007).

9. K. Jefimovs, J. Vila-Comamala, M. Stampanoni, B. Kaulich, and C. David, J. Synchrotron Radiat. 15, 106 (2008).

10. S. Gorelick et al., Microelectron. Eng. 87, 1052 (2010).

11. K. Jefimovs et al., Microelectron. Eng. 84, 1467 (2007).

12. P. Modregger, D. Lubbert, P. Schafer, and R. Kohler, Phys. Status Solidi A 204, 2746 (2007).

13. F. Zernike, Physica 1, 689 (1934).

14. C. Maurer, A. Jesacher, S. Bernet, and M. Ritsch-Marte, Opt. Express 16, 19821 (2008).

15. C. A. Larabell and M. A. Le Gros, Mol. Biol. Cell 15, 957 (2004). 